\title{
Brain segmentation unmasks association between body composition and central nervous system structures
}

\author{
Katarzyna Katulska', Mateusz Wykrętowicz', Tomasz Krauze², Jarosław Piskorski ${ }^{3}$ \\ ${ }^{1}$ Department of Radiology, Poznan University of Medical Sciences, Poland \\ ${ }^{2}$ Department of Cardiology - Intensive Therapy, Poznan University of Medical Sciences, Poland \\ ${ }^{3}$ Institute of Physics, University of Zielona Góra, Poland
}

\begin{abstract}
Introduction. Excess body fat is currently the major health problem. We suggest that marker of fatness like BMI or percent of body fat are associated with gray matter volume (GMV) or brain areas responsible for cognitive functions.

Material and methods. Study was performed in 89 healthy individuals (mean age 58 yrs, 51 women). For brain segmentation and GMV we used whole-brain, high-resolution 3D T1-weighted images. For assessment of the fat content as a proportion of total body mass a bio-impedance analyzer was used.

Result. GMV was correlated, significantly and negatively with age but was not associated either with BMI nor body fat content. Body fat content was significantly and negatively associated with hippocampus and thalamus. Conclusion. We demonstrate that brain segmentation was able to unmask the association between body fat content and brain structures particularly involved in cognitive function.
\end{abstract}

Key words: body fat, body mass index, gray matter volume, hippocampus, thalamus, brain segmentation.

\section{Introduction}

Excess body fat is currently a major health problem. Rising body weight in concert with hypertension and diabetes contributes to the development of cardiovascular complications such as myocardial infarction, heart failure or stroke $[1,2,3]$. It has also been shown that fat distribution between different compartments may affect brain structure and function [4]. Moreover, current research has suggested that high body mass index (BMI) may represent a risk factor for the development of cognitive decline or dementia $[5,6,7]$. In the present study we address the question whether a marker of obesity such as BMI or the percentage of body fat are associated with gray matter volume (GMV) or brain areas responsible for cognitive functions.

\section{Material and methods}

The study was performed on 89 healthy individuals (mean age 58 years, 51 women). None of the subjects were taking any medication. The Poznan University Ethics Committee approved the study protocol and a written informed consent was obtained from all participants.

Magnetic Resonance Imaging of the brain Magnetic Resonance Imaging (MRI) was performed using a 1.5-T magnet with a head 12-channel coil (Magnetom Avanto System, Erlangen Germany). We used axial and sagittal acquisition of T1-weighted, T2-weighted, PD, FLAIR and Diffusion Weighted Images (DWI). T1 and T2-weighted images were scanned 
with 4 millimeter slices, covering the whole brain. We used 230x230 field of view (FOV) and 256x256 scan matrix.

Whole-brain, high-resolution 3D T1-weighted images (Magnetization Prepared Rapid Gradient-Echo) were acquired for morphological analysis. The acquisition parameters were as follows: bandwidth $=190 \mathrm{~Hz} /$ pixel, flip angle $=7^{\circ}, \mathrm{TR} / \mathrm{TE} / \mathrm{TI}=2.73 \mathrm{~s} / 3.44 \mathrm{~ms} / 1 \mathrm{~s}$. This sequence was obtained to calculate grey matter volume (GMV) and brain segmentation.

Morphometric analysis of brain structure was completed with MRIcro Version 1.35 software (www. sph.s.edu/comb/rorden/mricro.html).

For detailed brain structure segmentation into volumes and surfaces, with the use of the state-of-the-art algorithms, the FreeSurfer Version 5.1 (Laboratory for Computational Neuroimaging, Athinoula A. Martinos Center for Biomedical Imaging) software package was used (http://surfer.nmr.mgh.harvard.edu), (Figure 1).

\section{Body fatness assessment}

For the assessment of the fat content as the proportion of a total body mass, a bio-impedance analyser (MC180MA, Tanita Corp USA) was used. Bio-impedance analysis was performed using multifrequency technology.

\section{Statistical analysis}

The results are expressed as mean \pm SEM, and Pearson's correlation coefficient was calculated. The statistical analyses were performed using MedCalc (MedCalc

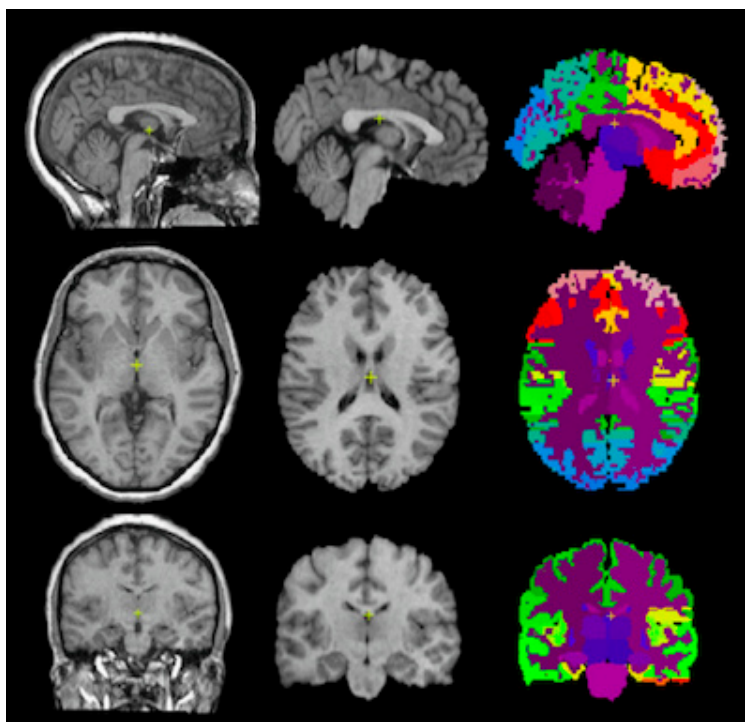

Figure 1. Representative maps of the white and gray brain matter in T1-weighted images. Segmentation of the brain
Software, Ostend, Belgium), with statistical significance set at $P<0.05$.

\section{Results}

Patients characteristics are presented in Table 1.

Table 1. Clinical characteristics of the study participants

\begin{tabular}{lc}
\hline \multicolumn{2}{c}{ Characteristic } \\
\hline Age (years) & $58 \pm 1$ \\
\hline M/F & $48 / 51$ \\
\hline BMI $\left(\mathrm{kg} / \mathrm{m}^{2}\right)$ & $26 \pm 0.4$ \\
\hline Body fat $(\%)$ & $27 \pm 1$ \\
\hline Cholesterol $(\mathrm{mg} / \mathrm{dl})$ & $210 \pm 4$ \\
\hline SBP $(\mathrm{mmHg})$ & $122 \pm 1$ \\
\hline $\mathrm{DBP}(\mathrm{mmHg})$ & $74 \pm 1$ \\
\hline
\end{tabular}

$M$ - male, F-female, BMI - body mass index, SBP - systolic blood pressure, DBP diastolic blood pressure
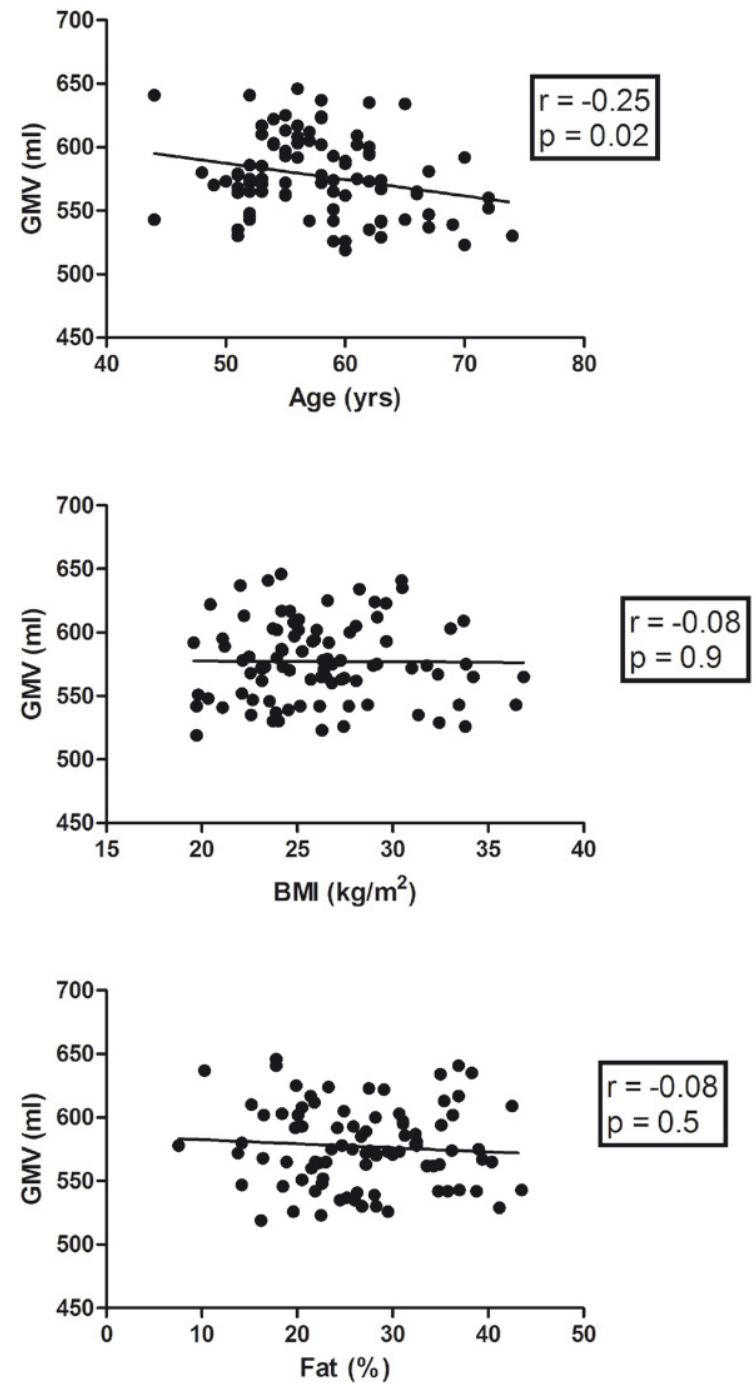

Figure 2. The association between gray matter volume, age, body mass index and percentage of body fat. GMV - gray matter volume, BMI body mass index 
Association between gray matter volume, measures of fatness and age.

In Figure 2, the correlation between gray matter volume, BMI and body fat content is presented. GMV was correlated, significantly and negatively with age $(r=-0.25, p=0.02)$ but was not associated either with BMI $(r=-0.08, p=0.9)$ or body fat content $(r=-0.08$, $p=0.5)$.

\section{Interaction between brain structures extracted} by segmentation and measures of fatness

The hippocampus and the thalamus were extracted with the use of FreeSurfer software. Body mass index correlated significantly and positively with body fat percentage $(r=0.5, p<0.0001)$, nevertheless a high degree of correlation does not mean that these measures could be regarded as interchangeable. As shown in Figure 3, both the hippocampus and the thalamus correlated significantly and negatively with age $(r=-0.37$, $p=0.0005 ; r=-0.29, p=0.009$, respectively). The hippocampus and the thalamus were not correlated with BMI ( $r=0.02, p=0.8 ; r=-0.12, p=0.2$, respectively). However, body fat content was significantly and negatively associated with both structures, namely the hippocampus and the thalamus $(r=-0.22, p=0.04$; $r=-0.24, p=0.03$, respectively).

\section{Discussion}

Apart from being an important risk factor for the development of diabetes, heart failure or stroke, obesity is thought to play a major role in cognitive decline and Alzheimer's disease. It has been demonstrated that Alzheimer's disease and obesity are both associated with brain volume decrease [7, 8]. Gustafson et al. [9] examined body mass index (BMI) in relationship to cerebral atrophy in a representative sample of women followed up from 1968 to 1992 as part of the population study. The authors were particularly interested in changes observed in temporal lobe since this area is highly susceptible to the effects of ischemia and other vascular insults to the brain. Moreover, it appears to be an early hallmark of Alzheimer's disease. Women with atrophy of the temporal lobe were, on average, by 1.1 to $1.5 \mathrm{~kg} / \mathrm{m}^{2}$ higher in BMI. Moreover, multivariate analyses showed that age and BMI were the only significant predictors of temporal atrophy. It is also important that there were no associations between BMI and atrophy measured at three other brain locations. Taki et al. [10] obtained brain MR images from 690 men and 738 women. Volumetric analysis revealed a significant negative correlation between $\mathrm{BMI}$ and the gray matter ratio (which represents the percentage of gray matter volume in the intracranial volume) in men (adjusting for age, lifetime alcohol intake, history of hypertension and diabetes mellitus), but not in women. Brain segmentation revealed that, in men, the regional gray matter volume of the bilateral medial temporal lobes, anterior lobe of the cerebellum, occipital lobe, frontal lobe, precuneus and midbrain showed significant negative correlations with BMI, while those of the bilateral inferior frontal gyri, posterior lobe of the cerebellum, frontal lobes, temporal lobes, thalami and caudate heads showed significant positive correlations with BMI. Thus, even these two articles demonstrated that body fat content estimated by BMI is to some extent associated with brain structure alterations. Moreover, it seems that in order to observe this correlation a large sample of subjects is required to increase the strengths of a statistical test applied to estimate such association. It needs to be noted that BMI is a measure of excess weight relative to height but not necessarily the excess adiposity. It is stressed by the fact that BMI in our study correlated only to some extent $(r=0.5)$ with body fat composition estimated by bioimpedance. We were also unable to observe any correlation between $\mathrm{BMI}$ and gray matter volume or white matter volume (data not shown). However, when we performed brain structure segmentation with the delineation of the thalamus and the hippocampus, both of these structures demonstrated association with body fat content but not with BMI. It is thought that the thalamus is involved in cognitive and perceptual function, while the hippocampus is involved in minute-to-minute cognitive processing including spatial information processing, temporal sequencing and formulating the relationships between objects in the environment [11, 12]. There are several reports with data indicating that body fatness may be associated with cognitive function. Yoon et al. [13] observed 250 subjects (mean age 60 years) to investigate a direct association between visceral adiposity and cognitive performance. It was concluded that high adiposity, particularly visceral adiposity, was associated with poor cognitive functioning in younger elderly persons. Interesting data indicate that under real-world stress higher body fat percentage may be associated with endocrine stress vulnerability, with consequences for deleterious cognitive performance [14].

Our study, although it involved a relatively small population, thanks to state-of-the-art brain segmentation used was able to unmask the association between 

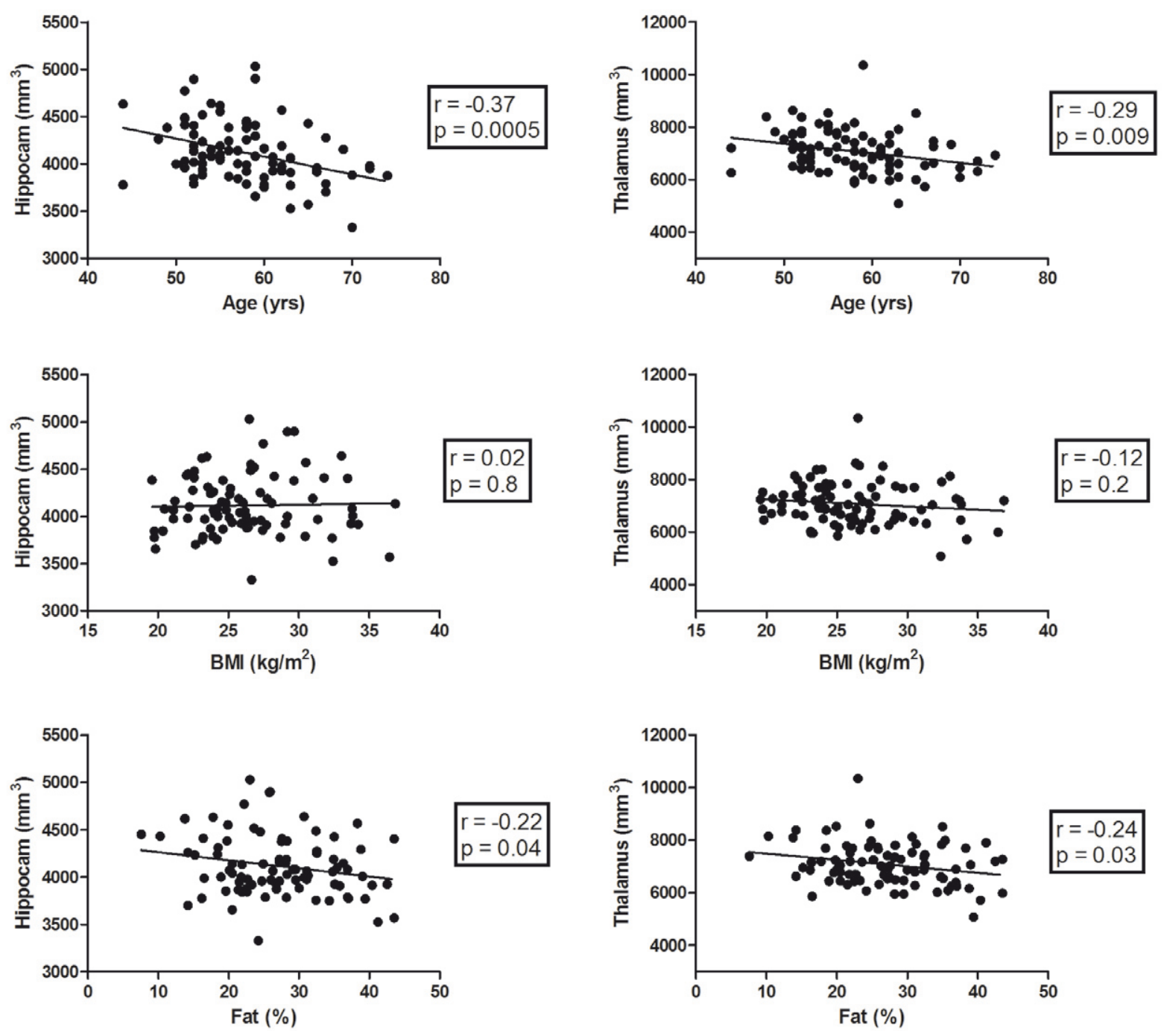

Figure 3. The association between volume of the hippocampus or the thalamus and age, body mass index or body fat. GMV - gray matter volume, $\mathrm{BMI}$ - body mass index

body fat content and brain structures particularly involved in cognitive function. The results of the study suggest that the improvement in the technique of MRI interpretation and delineation of structures of particular interest allow better insight into the pathophysiological link between body fat composition and brain arrangement.

\section{References}

1. Messerli FH. Cardiovascular effects of obesity and hypertension. Lancet. 1982;2:1165-8.

2. Manson JE, Colditz GA, Stampfer MJ, et al. A prospective study of obesity and risk of coronary heart disease in women. N Engl J Med. 1990;322:882-9.
3. Abbott RD, Behrens GR, Sharp DS, Rodriguez BL, Burchfiel CM, Ross GW, Yano K, Curb JD. Body mass index and thromboembolic stroke in nonsmoking men in older middle age: the Honolulu Heart Program. Stroke. 1994;25:2370-6.

4. Debette S, Beiser A, Hoffmann U, Decarli C, O'Donnell CJ, Massaro JM, Au R, Himali JJ, Wolf PA, Fox CS, Seshadri $\mathrm{S}$. Visceral fat is associated with lower brain volume in healthy middle-aged adults. Ann Neurol. 2010;68: 136-44.

5. Nourhashémi F, Deschamps V, Larrieu S, et al. Personnes Agées Quid. Body mass index and incidence of dementia:the PAQUID study. Neurology. 2003;60:117-9.

6. Chu LW, Tam S, Lee PW, et al. Late-life body mass index and waist circumference in amnestic mild cognitive impairment and Alzheimer's disease. J Alzheimers Dis. 2009; 17:223-32. 
7. Kanaya AM, Lindquist $K$, Harris TB, et al. Total and regional adiposity and cognitive change in older adults: the Health, Aging and Body Composition (ABC) study. Arch Neurol. 2009;66:329-35.

8. Gustafson DR, Steen B, Skoog I. Body mass index and white matter lesions in elderly women. An 18-year longitudinal study. Int Psychogeriatr. 2004;16:327-36.

9. Gustafson D, Lissner L, Bengtsson C, Björkelund C, Skoog I. A 24-year follow-up of body mass index and cerebral atrophy. Neurology. 2004;63:1876-81.

10. Taki Y, Kinomura S, Sato K, Inoue K, Goto R, Okada K, Uchida S, Kawashima R, Fukuda H. Relationship between body mass index and gray matter volume in 1,428 healthy individuals. Obesity. 2008;16:119-24.

11. Sweatt JD. Hippocampal function in cognition. Psychopharmacology. 2004;174:99-110.

12. Saalmann YB, Kastner S. Cognitive and perceptual functions of the visual thalamus. Neuron. 2011;28;71: 209-23.
13. Yoon DH, Choi SH, Yu JH, Ha JH, Ryu SH, Park DH. The relationship between visceral adiposity and cognitive performance in older adults. Age Ageing. 2012;41: 456-61.

14. Mujica-Parodi LR, Renelique R, Taylor MK. Higher body fat percentage is associated with increased cortisol reactivity and impaired cognitive resilience in response to acute emotional stress. Int J Obes. 2009;33:157-65.

\section{Correspondence address:} Katarzyna Katulska Department of Radiology Poznan University of Medical Sciences 49 Przybyszewskiego Stret 60-355 Poznań, Poland phone: +48 501698777 email: katarzyna_katulska@op.pl 